\title{
La Formación Profesional en Psicología y su Evaluación Nacional en México: un Estudio de Caso
}

\author{
Professional Education in Psychology and its National \\ Evaluation in Mexico: a Case Study
}

\author{
José Alfonso Jiménez Moreno * \\ Universidad Autónoma de Baja California, México
}

\begin{abstract}
El artículo presenta el problema de la formación profesional de los psicólogos en la Universidad Autónoma de Baja California (UABC) y su evaluación al término de sus estudios. A partir de las políticas institucionales que orientan la necesidad de conocer el desempeño de los egresados mediante el Examen General para el Egreso de la Licenciatura (EGEL) - prueba de alcance nacional que se utiliza como referente de competitividad-, se buscó la identificación de factores relacionados con los resultados de un grupo de egresados de psicología, así como indagar en sus condiciones de formación profesional dentro de la universidad. Mediante el enfoque metodológico de la teoría fundamentada y el uso de grupos focales, el análisis inductivo realizado permitió identificar cuatro categorías que sirvieron de sustento para la comprensión de este caso: razones para presentar el EGEL; apoyos y preparación para el examen; opinión sobre el examen; y, opinión sobre la formación. Se concluye la necesidad de valorar si la formación del psicólogo de la UABC debe orientarse o no hacia un estándar nacional, analizar la didáctica utilizada en los procesos de enseñanza y la aparente falta de exigencia en la formación profesional.
\end{abstract}

Palabras clave: Evaluación del estudiante; Formación profesional superior; Educación superior; Calidad de la educación; Psicología.

The article presents the problem of professional education of psychologists at the Autonomous University of Baja California (UABC) and its evaluation at the end of their studies. Based on the institutional policies that guide the importance of assess the performance of graduates through the General Test for Bachelor Graduation (EGEL) -test of national scope that serves as a benchmark for competitiveness-, this investigation focused on the identification of related factors with the results of psychology graduates, as well, to investigate the conditions of their professional education. With the use of the grounded theory as a methodological approach, and the use of focus groups, the inductive analysis carried out allowed the identification of four categories that served as support for the presentation of results: reasons for presenting the EGEL; supports and preparation for the exam; opinion about the test; and, opinion on their own education. The conclusion presents the need to analyze if the psychologist education should orient to a national standard, analyze the didactics used in teaching processes and the apparent lack of exigency on professional education.

Keywords: Student evaluation; Superior professional education; Higher education; Quality of education; Psychology.

\footnotetext{
*Contacto:

jose.alfonso.jimenez.moreno@uabc.edu.mx

Recibido: 14 de septiembre de 2018

$1^{\text {a }}$ Evaluación: 11 de octubre de 2018

issn: 1989-0397

$2^{\text {a }}$ Evaluación: 25 de octubre d 2018

www.rinace.net/riee/

Aceptado: $\quad 30$ de octubre de 2018
}

https://revistas.uam.es/riee 


\section{Introducción}

En la actualidad, el interés por identificar las competencias alcanzadas por los estudiantes de educación superior ha ido en aumento. En México esto es una práctica regular y, de manera particular, la Universidad Autónoma de Baja California (UABC) -institución de financiamiento público al noroeste de México- ha establecido una fuerte política orientada a conocer con precisión diversos indicadores que orienten su formación, ello con fines de retroalimentación de la educación profesional fomentada durante los estudios. Este interés está establecido en el Plan de Desarrollo Institucional 2015-2019 (PDI) (UABC, 2015), documento que rige la política y acciones estratégicas institucionales. Esta intención de la UABC de valorar la formación de los egresados se desarrolla bajo el contexto económico enfocado en la competitividad, en el cual es relevante conocer los resultados de los estudiantes a nivel universitario, al igual que su productividad, sus necesidades de formación y lo que realmente aprenden (Muñoz, 2007).

El interés de valorar el avance de las competencias de los egresados de formación profesional de la UABC permea a todos sus programas educativos. Para la valoración de estos elementos, además del trabajo de evaluación del aprendizaje que cotidianamente se realiza dentro del aula, la UABC hace uso del Examen General para el Egreso de la Licenciatura (EGEL), desarrollado por el Centro Nacional de Evaluación para la Educación Superior (CENEVAL). El CENEVAL es una asociación civil sin fines de lucro que desde hace más de 20 años se encarga de desarrollar instrumentos a gran escala sistematizados que permitan que las instituciones educativas públicas y privadas de México obtengan datos en relación con el logro de sus estudiantes (Gago, 2000). Actualmente existen 39 EGEL, uno para una disciplina distinta, de los cuales la UABC hace uso para identificar los indicadores de calidad en su formación, ello como un indicador de competitividad de su formación académica (Jiménez, 2017a).

Todas las unidades académicas de la UABC participan en la intención de identificar el logro de sus egresados, sin embargo, solo los programas en concordancia con los 39 EGEL del CENEVAL pueden obtener dicha información. Uno de los programas de mayor matrícula dentro de la UABC es la Licenciatura en Psicología, misma que participa de la aplicación del EGEL para conocer, año con año, el nivel de logro de sus estudiantes respecto al estándar nacional que establece el EGEL en Psicología (EGEL-PSI). El caso de la Licenciatura en Psicología es de interés en particular, ya que es el único programa educativo de la UABC que se ofrece en diversos campus con planes de estudio distintos. En ese sentido, los resultados de desempeño bajo este indicador resultan de especial atención.

$\mathrm{El}$ interés de la UABC y de sus académicos de conocer el logro de los egresados de diversas licenciaturas, así como la comprensión de los resultados en el EGEL son temáticas que actualmente han derivado en una serie de ponencias y publicaciones que han favorecido a la comprensión y crítica de la relación entre la formación profesional y el EGEL (Jiménez, 2017a, 2017b; Jiménez y Gutiérrez, 2017; Jiménez, Ojeda y Caso, 2018; Vázquez, Hirales y Botello, 2017). Frente a esta intención académica, y bajo el marco institucional que marca un interés explícito en los datos que arroja del EGEL sobre los egresados de la UABC (2015), el presente manuscrito muestra los resultados de una investigación que tuvo por objetivo identificar los elementos contextuales que los egresados psicología manifiesten como relevantes para la obtención de un resultado en particular en un EGEL. 
De manera particular, el análisis se centró en egresados que estudiaron en la Facultad de Ciencias Administrativas y Sociales (FACyS).

\section{La formación en Psicología en la UABC}

La Licenciatura en Psicología de la UABC se caracteriza por ser el único programa educativo de esta institución que tiene diferentes planes de estudio entre campus universitarios. Previo a revisar las características de cada uno de ellos, vale considerar que todos los programas de licenciatura de la UABC se fundamentan en un modelo por competencias, esto es relevante, ya que orienta la formación profesional de todas las áreas del conocimiento hacia la resolución de situaciones profesionales.

De manera particular, los objetivos y el perfil de egreso de la Licenciatura en Psicología del campus Tijuana, en su Plan de estudios 2012-2 (UABC, 2018b, p. 2) enuncia:

La formación profesional del Licenciado en Psicología implica un quehacer profesional ético, que ofrezca un servicio fundamentado en valores y actitudes congruentes, así como de un desempeño profesional basado en conocimientos y habilidades orientadas a la atención de necesidades humanas y demandas sociales de manera competente. Dichas características generales en la formación profesional del psicólogo dan sentido a la práctica profesional y fomentan el logro de las siguientes competencias:

$\checkmark$ Analizar el comportamiento humano de forma integral, desde distintos enfoques psicológicos, para identificar las diferencias individuales de forma ética y respetuosa hacia el individuo.

$\checkmark$ Analizar las relaciones interpersonales para diseñar estrategias de comunicación y modificación de la conducta que contribuyan a la solución de la problemática individual y psicosocial, a través de métodos y técnicas psicológicas con una actitud de apertura y respeto al otro.

$\checkmark \quad$ Evaluar las características psicológicas y de desempeño del recurso humano para promover la mejor expresión de sus potenciales personales, reflejados en el funcionamiento óptimo de las organizaciones, a partir del diagnóstico organizacional con legalidad y responsabilidad.

$\checkmark$ Aplicar estrategias y acciones psicopedagógicas a través de los instrumentos psicológicos, para incidir en todos los niveles del ámbito educativo de forma sensible y empática.

$\checkmark \quad$ Intervenir con técnicas psicológicas de evaluación y estrategias psicoterapéuticas en el cambio del comportamiento humano a partir de diversos enfoques psicológicos para la promoción de estilos de vida saludables, acorde al código ético del psicólogo.

El mapa curricular de esta formación se fundamenta en las áreas de ejercicio profesional: psicología general, educativa, organizacional, social, clínica, investigación y biológica. Este programa se aplica en las unidades Tijuana y Valle de las Palmas, ambas pertenecen al campus Tijuana.

Por su parte, el Plan de estudios 2012-2 de la Licenciatura en Psicología de los campus Ensenada y Mexicali establece lo siguiente (UABC, 2018a, p. 2):

El profesional de la Licenciatura en Psicología egresado de la UABC es competente para evaluar, diagnosticar, intervenir e investigar fenómenos y situaciones psicológicas desde una perspectiva científico-metodológica a nivel individual y grupal, en los contextos educativos, sociales, de la salud y organizacional; además de poseer valores éticos y actitudes profesionales como son la responsabilidad, organización, disciplina, compromiso, objetividad, empatía, eficacia y trabajo en equipo. El profesional de la Licenciatura en Psicología egresado de la $U A B C$ es competente para: 
$\checkmark$ Evaluar una situación o proceso psicológico de índole individual o colectiva, con metodologías cuantitativas y/o cualitativas (mixtas) propias de la disciplina, de forma responsable y comprometida, con el objeto de proporcionar referentes para la toma de decisiones y actuar en contextos regionales, nacionales e internacionales.

$\checkmark$ Diagnosticar el estado psicológico del individuo o colectivo que se valora, a través de la integración de la información disponible, para denominar el estado actual de la situación o fenómeno, en base a los lineamientos y referentes nacionales $e$ internacionales establecidos $y$ validados, con responsabilidad, respeto $y$ profesionalismo.

$\checkmark \quad$ Intervenir en situaciones o fenómenos psicológicos, con prácticas y procedimientos pertinentes, fundamentados desde el ámbito de la Psicología, tendientes a transformarlos o mejorarlos, de forma propositiva, con respeto y compromiso.

$\checkmark$ Investigar los fenómenos psicológicos que impacten en el desarrollo del ser humano, basándose en las teorías, técnicas y metodologías psicológicas, para la resolución de las demandas planteadas en los diferentes campos de la aplicación profesional, con rigor científico y responsabilidad.

Este programa fundamenta su estructura curricular en cuatro áreas, pero, a diferencia del que se ofrece en Tijuana, éste implica una organización en cuatro competencias que se consideran ejes para la formación del psicólogo: evaluación, investigación, diagnóstico e intervención. El programa se ofrece en el campus Ensenada, así como en Mexicali en sus dos unidades académicas (Mexicali y Guadalupe Victoria).

Si bien no es objeto de este manuscrito hacer una comparación curricular de la formación ofrecida en ambos campuses, vale destacar las diferencias entre ambos programas. Al menos con los elementos enunciados, se puede observar que el programa del campus Tijuana ofrece una formación con ejes basados en la división tradicional de la psicología, enfocándose en elementos de modificación conductual y psicoterapéuticos. En cambio, en Ensenada y Mexicali, la orientación se basa en competencias de ejercicio profesional (independientemente de las áreas de énfasis tradicionales de la disciplina), pero, además, se enuncian elementos claros del desarrollo humano.

La diferencia manifestada en ambos perfiles de egreso establece un antecedente importante para este trabajo, si bien superficial, permite valorar que la formación esperada en cada plan de estudios tiene diferencias importantes, que incluso se recalcan en las áreas de énfasis de la formación. El presente manuscrito se centra en los egresados de FCAyS, aunque valorar las diferencias de formación entre campus universitarios permite contextualizar los resultados, así como la necesidad de analizar posteriormente las particularidades de formación, mismos que probablemente pueden orientar la obtención de determinados resultados.

\section{El Examen General para el egreso de la licenciatura en psicología}

Este instrumento es una prueba de gran escala enfocada en la medición del logro educativo de los egresados de la Licenciatura en Psicología (y programas afines), con la finalidad de identificar si quienes lo presentan cuentan con las habilidades mínimas necesarias para un ejercicio profesional exitoso (CENEVAL, 2018). EL EGEL-PSI, al igual que el resto de los 38 EGEL, no se estructura con base en contenidos disciplinares que tradicionalmente suelen sustentar la formación profesional. Al pensar en la formación y ejercicio de la psicología tradicionalmente se hace referencia a áreas como: psicología clínica, educativa, 
social, organizacional, entre otras. El EGEL-PSI no se centra en las áreas de ejercicio, sino en las habilidades que se requieren del psicólogo en el ejercicio profesional en el contexto mexicano.

Como en cualquier ejercicio de evaluación, la delimitación conceptual de las áreas por valorar está supeditada a procesos basados, o bien en la literatura, o en procesos de definición colectiva. En el caso del EGEL-PSI, las áreas que lo constituyen las determina el Consejo Técnico, órgano colegiado coordinado por el CENEVAL con representantes de las diversas Instituciones de Educación Superior (IES) mexicanas que ofrecen la Licenciatura en Psicología (CENEVAL, 2018). La estructura del EGEL-PSI se muestra en el cuadro 1.

Cuadro 1. Estructura del EGEL-PSI

\section{ÁREAS Y SUBÁREAS DE LA PRUEBA}

NÚMERO DE REACTIVOS

- Evaluación psicológica

- Diagnóstico de comportamientos y procesos

- Evaluación de la intervención (eficacia y proyectos)

- Intervención psicológica

- Diseño de intervenciones

- Realización de intervenciones

- Investigación y medición psicológica

- Diseño de proyectos de investigación

- Realización de investigación básica o aplicada

- Diseño de instrumentos de medición y evaluación

Fuente: CENEVAL (2018).

Como se puede observar, la prueba no se estructura de manera conceptual, es decir, los ejes de este instrumento se basan en competencias o acciones del ejercicio profesional: evaluación, intervención e investigación y medición. Si bien se requeriría un análisis más a fondo, a simple vista la estructura de la prueba parece guardar correspondencia con la formación esperada del Plan de estudios que se imparte en Ensenada y Mexicali.

La comunicación de resultados del EGEL se muestran en tres niveles: Testimonio de Desempeño Satisfactorio (TDS), Testimonio de Desempeño Sobresaliente (TDSS), o bien, Sin testimonio (ST). El proceso de determinación de estándares y puntos de corte, así como los criterios para obtener un resultado en particular es definido ex profeso para cada EGEL y pueden ser consultados en CENEVAL (2018). Para efectos de este trabajo, baste considerar la segmentación del desempeño en estos tres niveles.

Existe un alto número de instituciones usuarias de este instrumento, en el 2017 se alcanzó un total de 348 IES y 11,309 sustentantes (CENEVAL, 2018). En el caso de la UABC, en ese mismo año 664 egresados de la UABC presentaron el EGEL-PSI. Em la figura 1 se muestran los resultados de los egresados de UABC en los últimos años.

La figura 1 muestra una comparación de los resultados de los sustentantes egresados de la UABC en sus tres campus: Ensenada, Tijuana y Mexicali respecto a los resultados a nivel nacional (es decir, el promedio de desempeño de todos los sustentantes del país). En los años presentados (2014 a 2017), los resultados de los egresados de Mexicali y Ensenada han sido, en lo general, más bajos que el promedio nacional. Por su parte, en 
Tijuana existe una mayor proporción de estudiantes en el llamado nivel Satisfactorio (TDS).

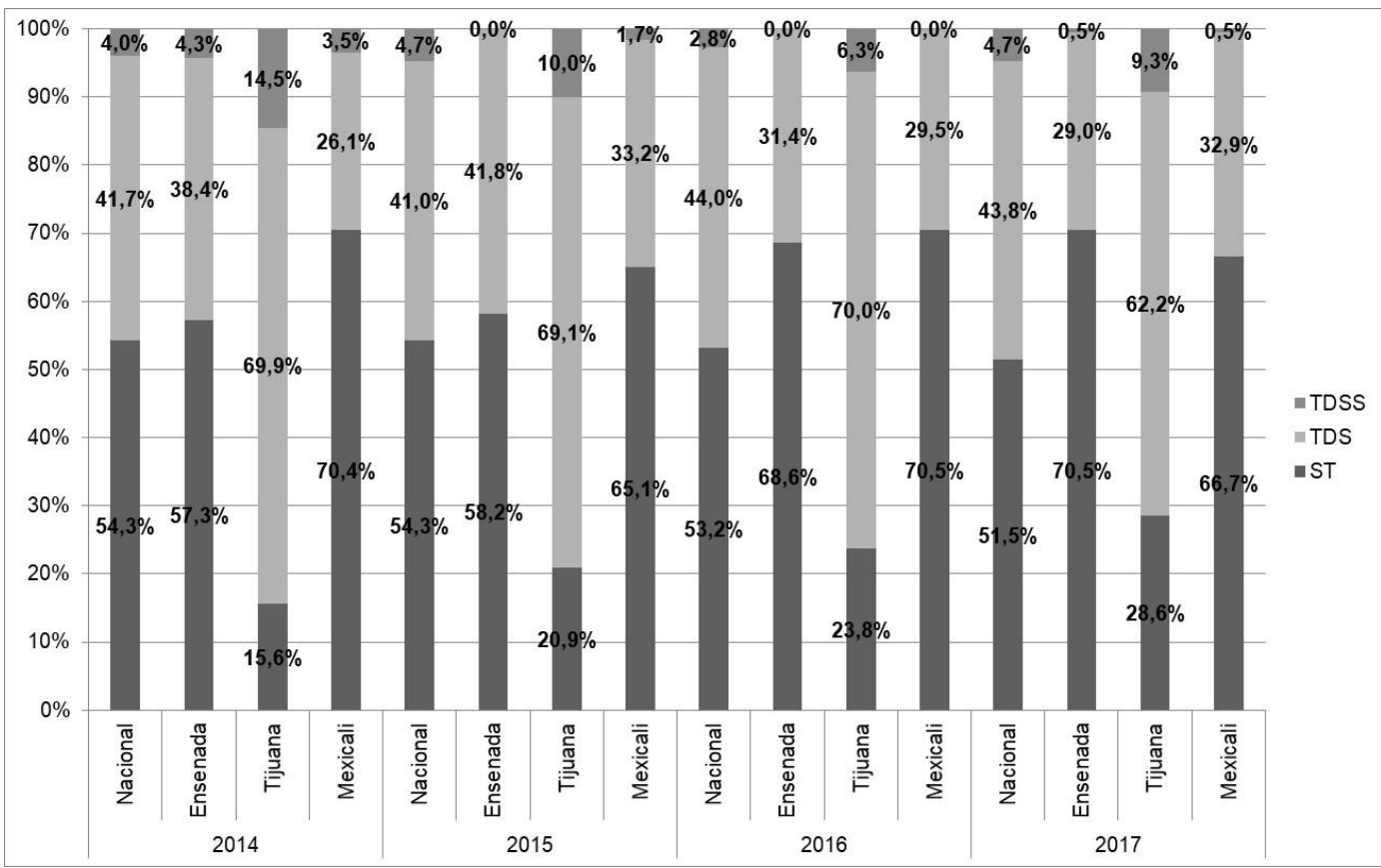

Figura 1. Resultados de los egresados de los diversos Campus de la UABC en el EGELPSI. 2014-2017

Fuente: Elaboración propia con datos de CENEVAL (2015, 2016, 2017, 2018).

Si bien en toda la UABC es posible analizar un análisis comparativo de los resultados de los egresados en el EGEL, resalta el caso de la Licenciatura en Psicología, dada la característica particular en la cual opera con dos programas distintos entre campus. En este sentido, bajo el interés de la institución de hacer uso de los resultados de los egresados en este indicador con fines de mejora de los procesos formativos, resalta la particularidad que entre 2014 y 2017, los resultados de los egresados del programa de psicología que se ofrece en los campus Mexicali y Ensenada difiera de los obtenidos en Tijuana.

El análisis de las condiciones de formación relacionada con una formación en psicología bajo dos planes de estudio distintos bajo el cobijo de un mismo modelo educativo es interesante, no solo como forma de conocer algunos factores que puedan estar relacionados con el resultado en el indicador, sino, sobre todo, por el análisis de las condiciones de formación que los egresados experimentaron.

\section{Revisión de literatura: investigaciones sobre condiciones asociadas a los resultados de la formación universitaria}

La identificación de elementos que permitan valorar los resultados de un proceso de formación profesional no es cosa sencilla, ya que está en función de la perspectiva conceptual que se adopte; sin embargo, existe una tendencia a considerar los resultados de estudiantes como un indicador de la calidad de la formación (Renault, Cortada y Castro, 2014). La búsqueda de análisis que consideren la relación de elementos de contexto de los 
estudiantes que permitan comprender los logros que obtienen en instrumentos a gran escala (como es el caso del EGEL) es algo que en el ámbito educativo se ha dado desde el llamado Informe Coleman (Coleman et al., 1966), el cual indicó que los factores externos a las escuelas tenían un gran impacto en el logro educativo, es decir, variables tales como la clase social y elementos culturales de la familia de proveniencia. A partir de ese antecedente se han generado diversas investigaciones para identificar cuáles son los factores que tienen incidencia en el logro educativo, esto a diferentes niveles de escolaridad.

De acuerdo con Tejedor (2003), Garbanzo (2007), Izar, Ynzuna y López (2011) y Gómez, Oviedo y Martínez (2011), las categorías que tienen mayor impacto en el rendimiento académico en estudios universitarios son: de identificación (género, edad, por ejemplo), psicológicas (aptitudes, motivación, hábitos de estudio), académicas (rendimiento previo, tipos de estudios cursados), pedagógicas (método de enseñanza, estrategias de evaluación) o sociofamiliares (estudios de los padres, situación laboral). Respecto a esta última categoría, el entorno familiar en el que se desenvuelve el estudiante universitario parece ser de gran relevancia, ya que la influencia de los padres y una sana convivencia son factores que pueden influir en el rendimiento académico (Guzmán y Pacheco, 2014; Martínez y Pérez, 2008;). A pesar de ello, la influencia de los elementos sociales y familiares en el rendimiento de los estudiantes universitarios es menor respecto a lo que puede suceder en otros niveles, dado que este nivel educativo implica que los estudiantes hayan superado ya una serie de filtros y los hacen una población más homogénea (Sánchez, 2007).

Incluso frente a esta relevancia del contexto familiar y social en el desempeño de los universitarios, existen diversas investigaciones que afirman que, en realidad, la trayectoria escolar de los estudiantes es lo que tiene mayor relevancia en su desempeño (Chaín, Cruz, Martínez y Jácome, 2003; Esguerra y Guerrero, 2010; Hernández, Márquez y Palomar, 2006; Izar, Ynzuna y López, 2011; Navarro, 2003; Ocaña, 2011; Tapasco, Ruiz y Osorio, 2016), esto se acentúa cuando nos referimos al desempeño de los egresados en el EGEL, donde el promedio de estudios de niveles previos tiene un valor predictivo en el resultado (Castillo, Izar y Espericueta, 2013). Sin embargo, otras investigaciones orientan que son elementos individuales, como la satisfacción del estudiante respecto a sus estudios (Becerra y Reidl, 2015; Kappe y Van der Flire, 2012; Sánchez, 2007; Van der Berg y Hofman, 2005) y la autorregulación para el estudio (Gaeta y Cavazos, 2016) lo que tiene mayor impacto en el rendimiento académico. A la par, también se desarrollan posturas que concluyen que son las características de la escuela formadora las que suelen tener un mayor efecto en las trayectorias y desempeño de los universitarios, más que las características individuales en sí, como lo podría ser la satisfacción propia respecto a sus estudios (Garbanzo, 2007; Shulruf, Hattie y Tumen, 2008; Toscano, Ponce, Margain y Vizcaíno, 2016); ello debido a que las condiciones institucionales que las universidades formadoras ofrecen, tales como apoyos económicos, servicio de tutoría y comunicación constante con los estudiantes, pueden tener un impacto favorable en el desempeño de los estudiantes (Peniche y Ramón, 2018).

Justo a partir de esta diversidad es necesario valorar la complejidad del fenómeno del desempeño de los universitarios. En ese sentido, la postura de Luna y Rosales (2014) nos permite ampliar esta visión, al afirmar que las prácticas educativas que tienen influencia en el rendimiento académico pueden variar dependiendo del nivel de análisis que se establezca, desde las políticas nacionales, los planes y programas de las instituciones 
formadoras, así como aquello relacionado con la práctica docente dentro del aula. Bajo esta lógica, la complejidad de un fenómeno como el que interesa en este manuscrito debe asumirse como tal, evitando establecer preponderancias conceptuales respecto a los elementos relacionados con el desempeño de los estudiantes de psicología de una universidad en condición de competitividad, como lo es la UABC. Sin embargo, vale resaltar la gran valía de la perspectiva de los egresados, la cual puede ser un insumo valioso para la mejora continua de la enseñanza universitaria, debido a su potencial como indicador de competencia profesional fomentada dentro de las instituciones formadoras (Rodríguez y Vieria, 2009).

En lo particular, respecto al uso del EGEL como medio de valoración del desempeño de los egresados universitarios y la identificación de los factores que se relacionan con sus resultados, Martínez y Pérez (2008) concluyen que el desempeño en el EGEL se ve asociado mayoritariamente con el promedio alcanzado en la formación profesional. En ese mismo sentido, Toscano, Ponce, Margain y Vizcaíno (2016) afirman que es la institución de procedencia la que tiene mayor incidencia, y, en segunda medida, el promedio obtenido en la formación (es decir, el desempeño académico mostrado en el trayecto universitario). Además de este par de investigaciones, existen algunos estudios en contextos de universidades públicas (Aguilar y Díaz, 2015; Barrera, Canepa y Santiago, 2016; Elías, Caldera, Reynoso y Zamora, 2016; García, Antonio y Gutiérrez, 2015; García y Rosales, 2016; Roblero y Orozco, 2015); sin embargo, no consideran las condiciones de formación expresados por quienes ya han presentado la prueba, salvo en el caso de la obtención de la opinión de los egresados respecto al instrumento en sí (Briceño, Rodríguez y Canto, 2011), a pesar de la importancia que su perspectiva puede aportar para retroalimentar la formación recibida.

Particularmente, respecto a estudios enfocados en la retroalimentación de la formación profesional de los psicólogos (sin tomar en cuenta aquellos resultantes de los esfuerzos institucionales denominados como "seguimiento de egresados"), existen diversos estudios con orientaciones centradas en la perspectiva de los empleadores (Ostrovsky y Di Doménico, 2007; Salazar, 2015), reflejando importantes necesidades de formación. Esta metodología también es relevante para la valoración de la formación profesional; sin embargo, ejercicios de análisis de la valoración profesional de psicólogos a través de la perspectiva de egresados en Latinoamérica, como los mostrados por Makrinov y Scharager (2005), Figueroa, Bernal y Andrade (2010) y Rodríguez y Serrano-García (2017) y muestran cómo las miradas de los egresados posibilitan el conocimiento de las condiciones institucionales que deben mejorarse en pro de la formación profesional.

\section{Método}

Con el uso del EGEL-PSI, al igual que con otros EGEL, CENEVAL ofrece a sus instituciones usuarias bases de datos de los sustentantes, en las cuales incluye los resultados junto con información de cuestionarios de contexto. Los informes anuales que el mismo CENEVAL (2018) pública incluyen orientaciones sobre las variables contextuales que mayor influencia tienen sobre los resultados en el EGEL. Sin embargo, a pesar que esta información puede ofrecer orientaciones para conocer los diferentes elementos relacionados con la formación de los egresados, se valora como limitada para identificar a fondo su experiencia universitaria, así como su perspectiva sobre el EGEL. Frente a ello, se tomó la decisión de considerar otras formas de acercamiento, 
particularmente debido al interés de identificar las particularidades de la formación en psicología dadas las diferencias entre campus. En concreto, se optó por trabajar bajo una perspectiva de estudio de caso (Stake, 2005); bajo este enfoque, la intención no es obtener resultados a partir de una muestra, sino orientar la comprensión de un caso preseleccionado, en este caso, un grupo de egresados de psicología de la FCAyS.

Con dicho antecedente, la presente investigación toma como método la teoría fundamentada (Glaser y Strauss, 1967). Bajo esta perspectiva, la valoración de categorías y códigos que permiten la interpretación del fenómeno observado requiere un trabajo analítico que suscita el desarrollo teórico a partir de los datos, más que centrarse en la verificación empírica de teorías ya determinadas, esto con el fin de generar teoría del fenómeno analizado. El desarrollo de este método se fundamenta esencialmente en el uso de técnicas cualitativas de recolección y análisis de datos. En el caso del presente manuscrito, se optó por el uso de grupos focales (Krueger y Casey, 2014), debido a que es una técnica que permite identificar en un grupo de personas con una experiencia común, en este caso, las particularidades de su formación. De esta manera, el uso de grupos focales bajo la lógica metodológica de la teoría fundamentada permitiría la comprensión de las particularidades de formación de los psicólogos egresados de la UABC, como un caso en particular, bajo un sistema analítico basado en la codificación de sus respuestas como insumo principal de generación de categorías teóricas sobre el fenómeno de su egreso.

La selección de los egresados participantes en los grupos focales se realizó bajo el acercamiento a la Facultad de Ciencias Administrativas y Sociales (FCAyS) de la UABC, unidad académica que ofrece la Licenciatura en Psicología, ubicada en el campus Ensenada de la universidad. Si bien hubiera sido deseable contar con grupos focales con egresados de psicología de los tres campus de la UABC, este primer acercamiento permitió delimitar un marco analítico-conceptual a partir de las respuestas de los egresados como un referente para trabajos posteriores más amplios. Con esa consideración, es menester tomar en cuenta la profundidad de los resultados, así como las limitaciones de generalización de los mismos.

La dirección de la FCAyS y su equipo permitieron el acercamiento a los egresados de psicología que participaron en el estudio. Como resultado de esta gestión, se contó con la participación de 10 egresados. Los criterios de selección de participantes se centraron en aquellos que hubieran presentado el EGEL, así como procurar la mayor diversidad posible en cuanto al sexo de los egresados. El cuadro 2 muestra un resumen de las características de los participantes.

A cada transcripción se le otorgó un código con el que se resumen si es egresado es hombre o mujer, su resultado en el EGEL, así como la sigla de su primer nombre, solo con fines de identificación. Los participantes se caracterizaron porque $90 \%$ de ellos obtuvo un resultado TDS o TDSS, mientras que solo uno no obtuvo testimonio. Por otra parte, solo una de ellas no estudió el plan de estudios 2012-2, vigente al momento de realizar el estudio. El desarrollo del grupo focal se realizó en las instalaciones de la FCAyS de Ensenada, se grabó el audio con la autorización escrita de los participantes y se realizó la transcripción correspondiente con fines de análisis. 
Cuadro 2. Egresados de la Licenciatura en Psicología de FCAyS participantes en el grupo focal

\begin{tabular}{cccccc}
\hline PARTICIPANTE & $\begin{array}{c}\text { CóDIGO DE } \\
\text { PARTICIPANTE }\end{array}$ & SEXo & $\begin{array}{c}\text { AÑo DE } \\
\text { EGRESO }\end{array}$ & $\begin{array}{c}\text { AÑo DE } \\
\text { PRESENTACIÓN } \\
\text { DEL EGEL }\end{array}$ & $\begin{array}{c}\text { RESULTADO } \\
\text { (ST, TDSS, } \\
\text { TDSS })\end{array}$ \\
\hline 1 & HDS-E & Hombre & 2017 & 2017 & TDS \\
2 & HDS-J & Hombre & 2016 & 2016 & TDS \\
3 & MDS-R & Mujer & 2017 & 2017 & TDS \\
4 & MDS-K & Mujer & 2017 & 2017 & TDS \\
5 & MDS-A & Mujer & 2017 & 2017 & TDS \\
6 & HDS-MA & Hombre & 2017 & 2017 & TDS \\
7 & MDS-D & Mujer & 2013 & 2013 & TDS \\
8 & HST-C & Hombre & 2017 & 2017 & ST \\
9 & MSS-M & Mujer & 2017 & 2017 & TDSS \\
10 & MDS-T & Mujer & 2017 & 2017 & TDS \\
\hline
\end{tabular}

Fuente: Elaboración propia.

Cuadro 3. Descripción de categorías y códigos utilizados

\begin{tabular}{|c|c|c|}
\hline CATEGORÍA & Código & DESCRIPCIÓN \\
\hline \multirow{3}{*}{$\begin{array}{l}\text { Razones para } \\
\text { presentar el } \\
\text { EGEL }\end{array}$} & Opción de titulación & $\begin{array}{l}\text { Refiere a las condicionantes institucionales de la UABC } \\
\text { que influyen en la presentación del EGEL, } \\
\text { particularmente como opción de titulación de los } \\
\text { estudiantes. }\end{array}$ \\
\hline & $\begin{array}{l}\text { Generación de } \\
\text { indicadores } \\
\text { institucionales }\end{array}$ & $\begin{array}{l}\text { Intención de la UABC para que los estudiantes } \\
\text { presentan el EGEL como medio de generación de } \\
\text { indicadores institucionales. }\end{array}$ \\
\hline & Reto profesional & $\begin{array}{l}\text { Es la manera en que los egresados manifiestan que el } \\
\text { EGEL representa un elemento de motivación } \\
\text { relacionada con los conocimientos adquiridos. }\end{array}$ \\
\hline \multirow{4}{*}{$\begin{array}{l}\text { Apoyos y } \\
\text { preparación } \\
\text { para el examen }\end{array}$} & $\begin{array}{l}\text { Cursos } \\
\text { propedéuticos }\end{array}$ & $\begin{array}{l}\text { Estrategias institucionales diseñadas para promover } \\
\text { resultados positivos en el EGEL, mediante cursos } \\
\text { dentro de la formación profesional. }\end{array}$ \\
\hline & Práctica profesional & $\begin{array}{l}\text { El conjunto de actividades de la formación profesional } \\
\text { para la aplicación del conocimiento en una unidad } \\
\text { receptora }\end{array}$ \\
\hline & $\begin{array}{l}\text { Estudio } \\
\text { independiente }\end{array}$ & $\begin{array}{l}\text { Estrategias de repaso y estudio realizadas por cada } \\
\text { egresado sin un marco o guía institucional formal. }\end{array}$ \\
\hline & Apoyo familiar & $\begin{array}{l}\text { Estrategias de soporte emocional y dinámicas familiares } \\
\text { que impactaron en la preparación de los egresados para } \\
\text { el EGEL. }\end{array}$ \\
\hline \multirow{2}{*}{$\begin{array}{l}\text { Opinión sobre } \\
\text { el examen }\end{array}$} & $\begin{array}{l}\text { Relación entre } \\
\text { formación y examen }\end{array}$ & $\begin{array}{l}\text { Refiere a la cercanía conceptual existente entre la } \\
\text { formación profesional recibida en la FCAyS-UABC y los } \\
\text { contenidos del EGEL-PSI. }\end{array}$ \\
\hline & Valoración personal & $\begin{array}{l}\text { Refiere al juicio general que los egresados hicieron sobre } \\
\text { el examen. }\end{array}$ \\
\hline \multirow{2}{*}{$\begin{array}{l}\text { Opinión sobre } \\
\text { la formación }\end{array}$} & $\begin{array}{l}\text { Formación teórica y } \\
\text { práctica }\end{array}$ & $\begin{array}{l}\text { Perspectiva de los estudiantes sobre la formación teórica } \\
\text { y práctica en psicología recibida en la FCAyS-UABC. }\end{array}$ \\
\hline & $\begin{array}{l}\text { Valoración de sí } \\
\text { mismos como } \\
\text { profesionistas }\end{array}$ & $\begin{array}{l}\text { Perspectiva de los estudiantes de sí mismos como } \\
\text { profesionistas, ello como resultado de su formación como } \\
\text { psicólogos egresados de la FCAyS-UABC. }\end{array}$ \\
\hline
\end{tabular}

Fuente: Elaboración propia.

Considerando la perspectiva metodológica de la investigación, se utilizó la técnica de análisis de inducción inductiva (Thomas, 2006; Saldaña, 2015). Es necesario aclarar que, dado que en el desarrollo de grupos focales se requiere del uso de una guía que permita la 
discusión entre los asistentes, ésta se realizó considerando aspectos que pudieran generar la discusión entre los asistentes en relación con su experiencia con el EGEL-PSI. En coincidencia con el enfoque metodológico del estudio, el énfasis analítico inductivo permitió la definición de los códigos.

Los códigos resultantes del análisis inductivo de la transcripción del grupo focal permitieron un acercamiento hacia la comprensión de las condiciones de preparación y las características circundantes a la aplicación del instrumento. Las categorías definidas respondieron a las respuestas de los egresados, más que a la clasificación mostrada en la literatura revisada; ello permitió, a partir de la generación de preguntas instigadoras en los grupos focales, la interpretación de las respuestas de los egresados al fenómeno de interés. A continuación se muestran los resultados expresados por los egresados en función de las categorías establecidas.

\section{Resultados}

Las perspectivas expresadas por los egresados permitieron las identificar algunas de las particularidades de formación en psicología y factores relacionados con los resultados en el EGEL, como se pretendía. Se muestran los resultados por cada una de las categorías analíticas utilizadas.

\subsection{Razones para presentar el EGEL}

Si bien dentro de la UABC permea una política de presentación del EGEL a través de las acciones promovidas por el PDI (UABC-2015), lo expresado por los egresados de psicología de la FACyS es que es una opción de titulación (además de examen profesional, memoria de presentación de servicio social, entre otras, UABC, 2006) que representa una relativa facilidad para la obtención del grado. Este elemento orilla a que la presentación del EGEL-PSI sea atractiva para ellos. Ello se observa en las siguientes opiniones:

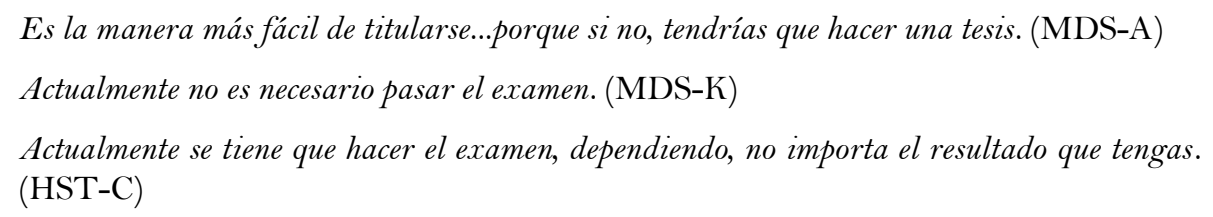

La política actual de la universidad promueve que todos los egresados presenten el EGEL. Esto se realiza con la finalidad de conocer el nivel de competitividad de los egresados, manifiesta a través de una prueba nacional a gran escala. Esta acción resulta atractiva para los egresados participantes como un medio que facilita los procesos de obtención del grado, que, de otra manera, implicaría el desarrollo de trabajos terminales, como una tesis. Resalta la opinión de HST-C, en el cual expresa que el resultado obtenido en el instrumento pudiera no tener relevancia, en función que la condición de obligatoriedad que la UABC establece implica solo presentar la prueba.

...Tienes que hacerlo [presentar el EGEL-PSI] y es preocupante, al menos en mi caso se me
hizo como muy importante que tienes que hacer y pasarlo, después te das cuenta de que no es
estrictamente necesario, que existen otras maneras graduarte. (MDS-K)

Por otra parte, los participantes también expresaron la importancia que la UABC ha hecho explícita sobre la necesidad de contar con indicadores educativos que reflejen la formación que la institución promueve en sus estudiantes. Tal como lo expresa la entrevistada: 
...Es un requisito porque la escuela lo necesita para ciertas estadísticas que le piden para saber cómo están los alumnos dentro de la escuela... nos decían que sí es importante el estudiar y pasarlo porque le da un buen prestigio a la escuela, pero no era un requisito pasarlo, era simplemente un dato estadístico. (MDS-A)

Esta opinión no fue generalizada entre los asistentes, sin embargo, resulta interesante que la institución transmita un mensaje sobre la relevancia que los resultados tienen en función de los intereses institucionales.

Por otra parte, se manifestaron algunas opiniones relacionadas con la posibilidad de valorar el EGEL-PSI como un reto profesional, más allá de la obligatoriedad institucional en su presentación:

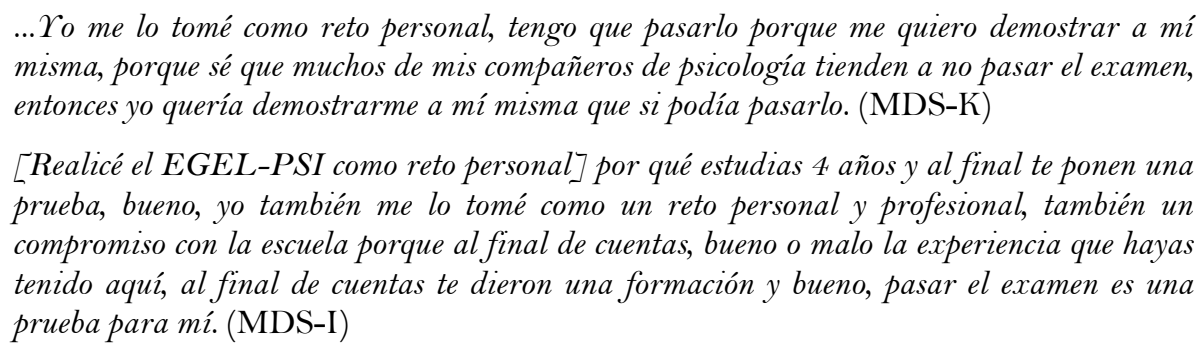

En el asumir el EGEL-PSI como un reto profesional se manifiesta una perspectiva sobre la formación recibida o los resultados bajos de la facultad en esta prueba. Resalta el hecho que ambas participantes obtuvieron resultados favorables en la prueba. Lo mismo sucede con los siguientes casos:

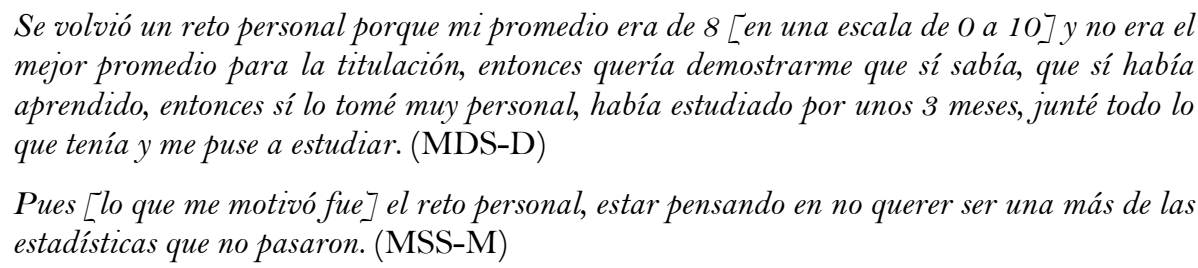

Las razones para presentar esta prueba, expresadas por los participantes, mantienen una influencia importante del discurso institucional, se manifiesta no solo en la obligatoriedad de presentar el EGEL-PSI, sino en la intención de generar estadísticas favorables para la universidad, así como tomar esta actividad como un reto personal y profesional relacionado con los resultados que la facultad ha obtenido en este instrumento, o bien, con el rendimiento académico propio.

\subsection{Apoyos y preparación para el examen}

Cuando los egresados estudiaban sus últimos semestres la institución hizo un esfuerzo para prepararlos para la presentación del EGEL-PSI; esto lo hizo a partir de una formación propedéutica.

A nosotros nos dieron una materia en octavo semestre llamada probabilidad y muestreo, no solamente era de psicología, o sea que también tocaban varios temas que venían dentro del examen, pero sí pusieron mucho énfasis a la estadística. (MDS-A)

En mi caso yo estuve en una optativa que era 'orientación' y la maestra nos dio la oportunidad de los últimos días de clases invitar a algunos maestros para que nos dieran algunas pláticas, que nos platicaron sobre cómo era la estructura del examen, invitó a algunos maestros a que nos dieran un repaso, pero era muy básico... [el curso se realizó] como tres o cuatro semanas antes del CENEVAL [EGEL-PSI], cuando invitaron a los maestros para que nos dieran un medio repaso. (MSS-M) 
Sí me sirvió bastante y todo lo que nos enseñaron en la optativa venía en el examen, no nos lo dieron como casos, pero al menos sí me enseñaron a ver sobre casos de estadística y preguntaban ciertas cosas que tenías que identificar dentro del caso [las preguntas que conforman el EGEL-PSI]. (MDS-R)

El programa de la Licenciatura en Psicología de la FCAyS ofrece a sus egresados una asignatura optativa en la cual, como los participantes manifiestan, se abordan temas que se asume estarán incluidas dentro del EGEL-PSI, asimismo, se da un repaso de los contenidos del plan de estudios, aunque también se abordan sugerencias de preparación:

To no asisti a las asesorías que otorgaron los de la mañana [los estudiantes del turno matutino], pero sí hubo una mini plática en el salón, llegaron algunos compañeros que habian hecho el examen en generaciones anteriores, y una de las cosas que salieron como conclusiones es que: 'no te presiones, confía en lo que sabes, relájate, ni trates de estudiar un día antes, desayuna bien, duerme bien, tú tienes el conocimiento, ahí está, tu confía en lo que sabes y ya. (MDS-I)

Además de este esfuerzo institucional, también se expresaron opiniones sobre la relevancia de la práctica profesional realizada. La práctica profesional, como se define en la Tabla 3, refiere a las actividades que los estudiantes de la UABC realizan fuera de la institución, poniendo en práctica los contenidos, métodos y procedimientos aprendidos dentro de las aulas; éstas se realizan dentro de unidades receptoras que suelen tener convenios formales con la universidad.

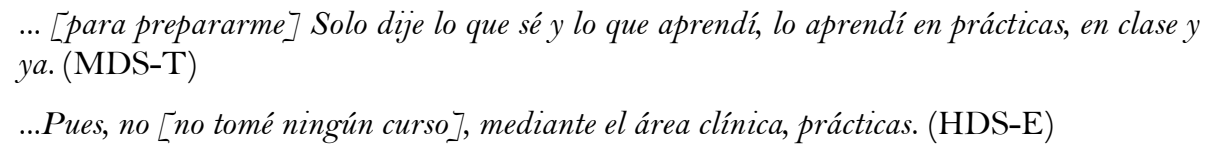

Además de las experiencias obtenidas en las prácticas profesionales, también se expresó la relevancia del estudio de manera independiente como medio de preparación para la presentación del EGEL-PSI.

\begin{abstract}
Sabía que [en el EGEL-PSI] venían muchas cosas o había escuchado que venían cosas que no daban en la carrera, regresaba de trabajar, entonces a eso me dediqué, de las tres áreas [del EGEL-PSI], lo dividi en las corrientes, en varios temas y subtemas, conforme empezaba a buscar información en Internet y en libros me daba cuenta de qué vimos y que no vimos, y ahi me enfoque, en lo que no había visto, confié en lo que ya había visto y no le di como peso, a metodología ya sabía que era mi 'coco' [le resultaba complicado] y me fui con una investigadora...en eso me enfoqué y sí me sirvió poquito. (MDS-D)
\end{abstract}

Por otra parte, como ha abordado la literatura, el apoyo familiar puede ser un elemento relevante dentro del desempeño académico de la formación universitaria (Sánchez, 2007; Martínez y Pérez, 2008; Guzmán y Pacheco, 2014). En el caso de los participantes, el apoyo familiar se dio de manera diferenciada.

Mi papá es maestro de investigación, entonces a mí me tocó la suerte de que mi papá fue el que me apoyó en todo el estudio de investigación y ellos acababan de presentar el EGEL, aunque de otra carrera, entonces de esa manera me apoyaron mucho. (MDS-A)

Mi mamá nada más me dijo "no te vayas a desvelar". (MDS-K)

Mi familia sabía, pero pues no fue un tema que se manejara de mucha importancia, era más relajado, "tu puedes, lo que aprendiste en la carrera es lo que vas a reflejar en el examen, todo tranquilo”. (MSS-M)

Pues tengo un bebé de dos años y sí podría decir que ese tiempo no fue mío, mi esposo me apoyaba, como estaba estresada, me dormía tarde, me despertaba a las 3 horas y así, así que mi esposo fue el que se hizo cargo de todo. (MDS-D) 
En los apoyos utilizados para la preparación del examen resalta el interés institucional por obtener resultados favorables, concretizado en una asignatura optativa orientada a reforzar elementos clave de la formación profesional bajo la óptica del EGEL-PSI. Si bien también hubo manifestación de apoyo familiar, preparación individual y el aprovechamiento de las prácticas profesionales, el énfasis en el interés institucional fue notable.

\subsection{Opinión sobre el examen}

Considerando los intereses de presentación del EGEL-PSI y los apoyos recibidos para sustentar este examen, los participantes manifestaron una postura interesante respecto a la opinión que les merece esta prueba. Algunos de los comentarios se centraron en la relación entre el EGEL-PSI y la formación recibida.

Bueno, total, son 4 años invertidos y algo de conocimiento sí tengo, porque hubo práctica, hubo conocimiento, hubo apoyo con los maestros'. Entonces, de alguna manera, fue como que solté esa parte, pero al principio sífue algo tenso para algunos de nosotros porque nos dimos cuenta que no sabíamos nada y cuando llegamos allá al examen, aparte de que fue cansado...también venían muchas cosas que hicimos en práctica como había cosas que no habíamos visto durante la carrera. (MDS-R)

Sí, honestamente, en el CENEVAL [el EGEL-PSI] sí venía, recuerdo muchas partes que no venían o no se vieron en la institución, en el área de investigación, de estadística, pero en esa parte tuve la fortuna de apoyarme en las investigaciones, agarré ese conocimiento, recordarlo y plasmarlo, pero sí es cierto, obviamente el CENEVAL, bueno, la carrera no está en seguimiento con el CENEVAL, porque tengo entendido que viene de la UNAM [Universidad Nacional Autónoma de México] o de México [Ciudad de México], por allá. (HDS-J)

En estas perspectivas se observa cómo los participantes valoran cierta distancia entre el EGEL-PSI y su formación profesional. Manifiestan que algunos contenidos del examen sí fueron revisados dentro de la formación, sin embargo, la falta de relación directa es notoria también.

Pero lo que sí me di cuenta que mientras hacía el examen me vinieron muchos recuerdos de clases, de muchos profesores que ponían sus clases en forma de casos, entonces la preparación que me dio la universidad años atrás fue lo que me ayudó a pasar. (MDS-K)

Paradójicamente, la misma participante manifestó también la falta de relación entre las formas didácticas experimentadas en la FCAyS y el tipo de preguntas que se abordan con el EGEL-PSI:

A mí lo que me hubiera gustado ver más en la carrera, para haberme sentido más preparada en la prueba, era que nos prepararon en un sentido de la vida real, porque muchas de las clases es memorizar, 'qué es esto', 'para qué sirve', 'cómo funciona', 'quién lo dijo', etc.; y la prueba, el EGEL, es mucho en la vida real, te presentan estos casos, con estas condiciones, ¿̇cómo lo vas a resolver? (MDS-K)

En ese mismo sentido, uno de los participantes afirmó lo siguiente:

Entonces pienso que el plan de estudios sí se queda muy corto en relación a ese examen. (MDSA)

Concuerdo mucho con lo que dice HDS-J, en lo que dice que lo que viene en el examen viene de la práctica, claro que no todos tienen la misma experiencia en las prácticas profesionales como en el caso de HDS-J y MSS-M, y la verdad yo estoy súper contento con el EGEL, mucho de lo que viene lo pude responder por eso, creo que un $40 \%$ fue gracias a las prácticas y un $60 \%$ gracias a la universidad. (MDS-A) 
Los participantes no manifestaron inconformidad con la prueba respecto a la posible falta de correspondencia entre la formación y los contenidos del EGEL-PSI, sin embargo, se manifiesta con claridad que la formación recibida no guarda estrecha relación con lo que se valora en su egreso y que con lo cual se determina su titulación.

La inconformidad se manifestó al hablar sobre la opinión general que tienen sobre el EGEL-PSI, particularmente en lo que refiere a su duración, dado que es una prueba que se aplica en un lapso de ocho horas en dos sesiones de cuatro horas cada una.

El tiempo, el tiempo que nos dieron para hacer el examen, como dice la compañera, ocho horas para estar ahí es demasiado, la segunda parte se me hizo súper cansadísima, me quedé dormida. (MDS-K)

Pues ya había comentado que estaba muy pesado el examen, la verdad el tiempo es muy exagerado...no puedes mantener la atención por tanto tiempo, la verdad es un factor que influye muchísimo en cómo contestas el examen, la tensión que se siente en el ambiente, los asientos incómodos, son muchos factores que en el momento afectan, aunque parezca que no. (MSS-M)

Además de la percepción sobre lo pesado que resulta contestar una prueba de ocho horas de duración, los participantes manifiestan la falta de relación que éste tiene con su formación profesional; los egresados argumentan la necesidad de articulación.

\subsection{Opinión sobre la formación}

Hasta ahora, los resultados han permitido valorar cómo las intenciones institucionales se manifiestan en las intenciones de presentación del EGEL-PSI, en los apoyos para la presentación de la prueba, así como la relación entre la formación y la prueba, todo ello bajo la perspectiva de los egresados participantes. Sin embargo, resulta valioso hacer mención de la visión que los egresados expresaron respecto a la formación recibida dentro de la FCAyS-UABC. Al respecto, se manifestaron opiniones respecto a la falta de formación práctica y falta de exigencia.

Creo que UABC debería de prestar más atención, porque en las clases, es mucho memorizar, exámenes, memoriza este concepto, relacionar cosas, pero creo que serviría mejor para formar mejores psicólogos y para un filtro, porque nadie puede negarlo, salen psicólogos como pan horneado del supermercado, salen de todos lados, salen demasiados... Siento que en realidad los maestros que a mí me impartieron clases, tienen todas las herramientas para formarnos como buenos psicólogos, pero sí, creo que UABC no exige la institución como tal, los tiempos de clases creo que hace falta mucha práctica, está bien que nos enseñen la teoría base para que formen un buen psicólogo, pero qué hago con esa teoría, creo que hacen falta más espacios para poder aplicar esa teoría, porque obviamente hay gente preparada. (MDS-A)

Les falta laboratorios para desarrollarnos nosotros, los alumnos que sí nos interesan, tiene demasiados alumnos... hay muchos alumnos mediocres que vienen aquí no sé si por costumbre, que no les importa... La universidad...y a tiene la costumbre de que a los alumnos no les importa...es lo que quiero creer que tiene UABC y su perspectiva tiene que cambiar y debería de haber más filtros, debería tener más filtros porque hay alumnos que nos les interesa estar aqui. (HDS-J)

Lo que yo he notado que [a las autoridades de la UABC] les gusta manejar mucho estadísticas pero no escuchan las exigencias de los empresarios hacia que se enfoquen más en adecuar esa parte de los contenidos que ven los alumnos durante su formación profesional, porque muchas veces lo que estás viendo no es lo mismo que se ven en las empresas, o no te lo vas a topar en la vida real, entonces creo que es pura pantalla [pantomima], no escuchan las peticiones de los empresarios que son los que te van a dar el trabajo allá afuera. (MDS-T) 
En estas opiniones se manifiesta cómo los participantes perciben poca exigencia en la formación, así como falta de correspondencia con actividades profesionales fuera del aula, e incluso con las necesidades del sector laboral. Además, se presentaron expresiones que manifestaban la opinión de la formación en términos culturales.

La UABC no te enseña a creérsela, no tiene esa cultura de decir: 'tú vas a ser un profesional, vas a ser un psicólogo, alguien allá afuera'; esa cultura tal vez no existe dentro de la universidad. Honestamente yo me siento muy identificado con la escuela, 'soy cimarrón', como dicen, tengo dos carreras aquí pero sí siento que la UABC es más de inculcar esa parte de 'no pasa nada. (HDS-J)

Dentro del grupo focal también se manifestaron opiniones relacionadas con la valoración sí mismos como profesionistas:

Cuando vi el 'sin testimonio' [el resultado en el EGEL], me sentí mal y muy mediocre, sentí como si esos 4 años en la carrera no valieran para nada y no puedo generar pensamientos positivos hasta la fecha, pero he buscado talleres, diplomados, en el área en que me gusta y sé que soy bueno en esa área y sé que por no pasar no puedo ejercer, porque sé que soy bueno y tengo vocación de psicólogo que creo que es lo más importante, que no muchos tienen. (HSTC)

Esta opinión es relevante, ya que las expectativas institucionales que relacionan el ejercicio profesional con el resultado en el EGEL-PSI (manifestada transversalmente en toda la discusión en el grupo focal) son valoradas en las razones para presentar el examen, en el apoyo para su preparación, en la relación con la formación y en la valoración propia del desempeño profesional. Esta perspectiva fue manifestada por el participante que no obtuvo un resultado favorable en la prueba; el resto de los participantes no manifestaron tanta relevancia al EGEL como indicador de su valía profesional, aunque, como se ha observado, se le refiere como relevante, al punto que se sugiere que la UABC oriente su formación hacia la perspectiva de la psicología que mantiene esta prueba.

\section{Discusión y conclusiones}

El presente trabajo se orientó inicialmente a identificar los factores que los egresados de la Licenciatura en Psicología de la FCAyS-UABC participantes en el estudio establecen como importantes para el resultado obtenido en el EGEL-PSI, sin embargo, en el desarrollo de la investigación, el trabajo permitió la identificación de elementos relacionados con la visión respecto a este instrumento y la preparación profesional de algunos estudiantes de psicología.

La revisión de la literatura ofreció la oportunidad de identificar elementos de relevancia que tradicionalmente se han relacionado con el desempeño académico de estudiantes universitarios. Resalta el apoyo institucional, el apoyo familiar, así como la trayectoria académica de los estudiantes. Para efectos de este trabajo, al utilizar el enfoque metodológico de la teoría fundamentada (Glaser y Strauss, 1967), se mantuvo abierta la posibilidad de identificar otros factores que los actores mismos identifican como importantes para efectos de la determinación del resultado en un estándar nacional. De esta manera, los egresados participantes en el estudio permitieron la identificación de cuatro categorías que permiten avanzar en esta línea de investigación: a) razones para presentar el EGEL, b) apoyos y preparación para sustentarlo; c) opinión sobre el examen; y, d) opinión sobre la formación. 
Dado que en UABC, como en muchas otras IES en México, la presentación del EGEL es una política vigente, que ha llevado al establecimiento de mecanismos que relacionan el resultado de la prueba con el egreso de la institución o la obtención del grado (Jiménez, 2017b). Los participantes en el estudio muestran cómo el interés institucional no se concreta únicamente con presentar el examen, sino que hace evidente la intención de los estudiantes de graduarse pronto; en esta intención, el EGEL-PSI funciona como un mecanismo óptimo para ello. Pero, además, que los egresados muestran la posibilidad que este interés institucional se deba a la necesidad de generar indicadores, y no a la intención de retroalimentación a la formación profesional, como se expresa en el marco institucional referido.

Respecto a los apoyos y preparación para sustentarlo, el interés institucional de obtener resultados favorables se manifiesta en la preparación de una asignatura optativa en la cual se resuman las generalidades de la formación en psicología, pero, además, de acentuar la formación en los aspectos en los que el EGEL-PSI hace énfasis. La inclusión de estas intenciones en el currículum refiere al interés institucional de reflejar el nivel de competitividad que manifiesta en su PDI (UABC, 2015). Resalta también cómo el apoyo familiar se manifiesta cómo lo hace la literatura (Sánchez, 2007), en el sentido que parecen una formación más homogénea que en otros niveles educativos.

Sobre la opinión respecto al examen, los sustentantes asumen la relevancia del EGEL-PSI como elemento de valor de la formación profesional, inculcado fuertemente por la actual política institucional. Se manifiestan en la necesidad de reorientar la formación profesional del psicólogo egresado de la FCAyS-UABC hacia el modelo de resolución de problemas de la vida profesional ofrecido por el EGEL-PSI. Resaltan la necesidad de ofrecer una formación que trascienda la perspectiva conceptual y teórica. Esta perspectiva de los egresados es importante, sobre todo al considerar la aparente relación del Plan de estudios que se ofrece en Ensenada y Mexicali respecto a la estructura del EGEL-PSI, además del modelo educativo universitario basado en competencias. Los resultados de estos dos campus son poco favorables respecto al estándar nacional y lo observado en el campus Tijuana, esto puede relacionarse con lo mencionado por los participantes, que valoran su formación ajena a la estructura del EGEL-PSI, orientada fuertemente hacia el ejercicio profesional y no a la repetición teórica.

Esto se relaciona estrechamente con la opinión sobre la formación. Como lo comentan los egresados, parece que la FCAyS-UABC es poco exigente respecto a la formación que ofrece en el ámbito de la psicología. Más allá de los contenidos curriculares, la práctica profesional o el interés propio es lo que apoya la obtención de un resultado en particular. No debe menospreciarse la perspectiva expresada por el participante que no obtuvo un resultado favorable, quien manifestó claramente una valoración poco favorable sobre su ejercicio profesional.

Los elementos observados a través de este ejercicio permitieron identificar otros elementos más allá de la perspectiva tradicional de los factores asociados con un resultado en un instrumento en particular. La voz de los egresados facilitó identificar la operación concreta de las políticas institucionales relacionadas con el fomento de la competitividad y la intención de los egresados de tener una formación menos teórica y mayormente orientada hacia la forma ofrecida por el estándar nacional de evaluación de la formación psicológica. Con la información generada de este caso en particular, es factible desarrollar más estudios que permitan orientar: a) si la formación debe o no estar orientada hacia un 
estándar nacional; b) si desea mantener procesos que favorecen la facilidad de titulación (independientemente del resultado que se obtenga en las evaluaciones); c) la didáctica utilizada en sus procesos de formación, ya que los egresados valoran con mayor relevancia a la experiencia generada en las prácticas profesionales que a los contenidos revisados en las aulas, elemento que debe relacionarse con el modelo educativo que da sostén el quehacer universitario; d) la aparente falta de exigencia en la formación profesional. No es menor la consideración sobre cómo las políticas institucionales, que resaltan el resultado del EGEL-PSI como valor del ejercicio profesional pueden impactar en la perspectiva de los egresados sobre su valor como profesionistas, aun cuando el EGEL-PSI no esté del todo relacionado con la formación recibida.

La principal limitación de este estudio se basa en la consideración de una sola unidad académica. La perspectiva de considerar un caso en aislado (Stake, 2005) permitió el conocimiento de las condiciones de formación profesional del psicólogo en esta facultad; sin embargo, el entendimiento de las condiciones de formación será mucho más robusto en medida que se involucre a otras unidades, particularmente aquellas pertenecientes al campus Tijuana, donde los egresados obtienen mejores resultados que en Mexicali y Ensenada, y donde aparentemente el Plan de estudios guarda menor correspondencia con la estructura del EGEL-PSI. A pesar de esta limitación, el enfoque metodológico utilizado permite tener un primer acercamiento teórico sobre algunas particularidades en la formación profesional en una universidad, cuyo interés en fomentar la competitividad es explícito; pero, además, vale resaltar la necesidad de identificar las condiciones de formación y su relación con las evaluaciones que se realizan a los estudiantes.

\section{Agradecimientos}

Este manuscrito se realizó gracias al financiamiento del Programa para el Desarrollo Profesional Docente para el Tipo Superior (PRODEP) de la Secretaría de Educación Pública, México, Folio: UABC-PTC-661. Asimismo, se agradece el apoyo del Dr. Sergio Cruz Hernández, Director de la Facultad de Ciencias Administrativas de la Universidad Autónoma de Baja California, para el desarrollo de esta investigación, así como la participación de Dania Jetzalinne Ojeda Osuna como apoyo en el desarrollo y transcripción de la información de los grupos focales.

\section{Referencias}

Aguilar, R. y Díaz, J. (2015). Desempeño histórico de estudiantes en el EGEL-ISOFT: El caso del primer programa de Ingeniería de Software en México. Educación y Ciencia, 4(44), 83-97.

Barrera, R., Canepa, A. y Santiago, J. (2016). Desempeño de egresados de tecnologías de información en el examen general de egreso. Revista Iberoamericana para la Investigación y Desarrollo, 7(13), 497-513. https://doi.org/10.23913/ride.v7i13.261

Becerra, E. y Reidl, L. (2015). Motivación, autoeficacia, estilo atribucional y rendimiento escolar de estudiantes de bachillerato. Revista Electrónica de Investigación Educativa, 17(3), 79-93.

Briceño, S., Rodríguez, J. y Canto, P. (2011, noviembre). El EGEL visto por sus sustentantes: significados asociados a la prueba y al TDSS. Comunicación presentada en el XI Congreso Nacional de Investigación Educativa. Recuperado de: http://www.comie.org.mx/congreso/memoriaelectronica/v11/docs/area_16/1277.pdf 
Castillo, A., Izar, J. y Espericueta, D. (2013). Correlación entre trayectoria académica y el examen nacional de egreso de la licenciatura. CPU-e. Revista de Investigación Educativa, 17, 172-188. https://doi.org/10.25009/cpue.voi17.427

Centro Nacional de Evaluación para la Educación Superior (CENEVAL). (2015). Informe anual de resultados 2014. Examen General para el Egreso de la Licenciatura en Psicología (EGEL-PSI). Ciudad de México: CENEVAL.

Centro Nacional de Evaluación para la Educación Superior (CENEVAL). (2016). Informe anual de resultados 2015. Examen General para el Egreso de la Licenciatura en Psicología (EGEL-PSI). Ciudad México: CENEVAL.

Centro Nacional de Evaluación para la Educación Superior (CENEVAL). (2017). Informe anual de resultados 2016. Examen General para el Egreso de la Licenciatura en Psicología (EGEL-PSI). Ciudad México: CENEVAL.

Centro Nacional de Evaluación para la Educación Superior (CENEVAL). (2018). Informe anual de resultados 2017. Examen General para el Egreso de la Licenciatura en Psicología (EGEL-PSI). Ciudad México: CENEVAL.

Chaín, R., Cruz, N., Martínez, M. y Jácome, N. (2003). Examen de selección y probabilidad de éxito escolar en estudios superiores. Estudio de una universidad pública estatal mexicana. Revista Electrónica de Investigación Educativa, 5(1).

Coleman, J., Campbell, E., Hobson, C., McPartland, J., Mood, A., Weinfeld, F. y York, R. (1966). Equality of educational opportunity. Washington, DC: National Center for Educational Statistics.

Elías, C., Caldera, J., Reynoso, O. y Zamora, M. (2016). Variables asociadas al rendimiento en el Examen General para el Egreso de Licenciatura. El caso de Psicología. Revista de la Educación Superior, 45(180), 75-88. https://doi.org/10.1016/j.resu.2016.06.008

Esguerra, G. y Guerrero, P. (2010). Estilos de aprendizaje y rendimiento académico en estudiantes de psicología. Revista Diversitas-Perspectivas en Psicología, 6(1), 97-109. https://doi.org/10.15332/s1794-9998.2010.0001.07

Figueroa, S., Bernal, B. y Andrade, C. (2010). Evaluación de un programa mexicano de maestría en psicología desde la perspectiva del egresado: un estudio sobre los indicadores de calidad. Revista de la Educación Superior, 39(1), 23-41.

Gaeta, M. y Cavazos, J. (2016). Relación entre tiempo de estudio, autorregulación del aprendizaje y desempeño académico en universitarios. CPUE-e. Revista de Investigación Educativa, 23, 142-166. https://doi.org/10.25009/cpue.voi23.2166

Gago, A. (2000). El CENEVAL y la evaluación externa de la educación en México. Revista Electrónica de Investigación Educativa, 2(2).

Garbanzo, G. (2007). Factores asociados al rendimiento académico en estudiantes universitarios, una reflexión desde la calidad de la educación superior pública. Revista Educación, 31(1), 4363. https://doi.org/10.15517/revedu.v31i1.1252

García, J., Antonio, P. y Gutiérrez, M. (2015). Estilos de aprendizaje y su relación con el instrumento EGEL-CENEVAL. Revista de Estilos de Aprendizaje, 8(16), 211-250.

García, R. y Rosales, F. (2016). Análisis de resultados del Examen General de Egreso CENEVAL (EGEL-CENEVAL) de la División de Ciencias Económicas y Sociales del 2012 al 2015 de la Universidad de Sonora Campus Caborca. Invurnus, 11 (1), 22-28.

Glaser, B. y Strauss, A. (1967). The discovery of grounded theory: strategies for qualitative research. Nueva York, NY: Aldine Publishing. 
Gómez, G., Oviedo, R. y Martínez, E. (2011). Factores que influyen en el rendimiento académico del estudiante universitario. Tecnociencia Chihuahua. 5(2), 90-97.

Guzmán, R. y Pacheco, M. (2014). Comunicación familiar y desempeño académico en estudiantes universitarios. Zona Próxima, 20, 79-91.

Hernández, J., Márquez, A. y Palomar, J. (2006). Factores asociados con el desempeño académico en el EXANI-I. Zona Metropolitana de la Ciudad de México. Revista Mexicana de Investigación Educativa. 11(29), 547-581.

Izar, J., Ynzunza, C. y López, H. (2011). Factores que afectan el desempeño académico de los estudiantes de nivel superior en Rioverde, San Luis Potosí, México. Revista de Investigación Educativa, 12. https://doi.org/10.25009/cpue.voi12.50

Jiménez, A. (2017a). El modelo educativo de la UABC y su correspondencia con el EGEL: discursos orientados hacia la calidad, competencia y rendición de cuentas. Debates en Evaluación y Currículum, 3(3).

Jiménez, A. (2017b). La evaluación de los egresados de formación profesional en México: Reflejo de la implementación de la política de competitividad en la Educación Superior. Archivos Analíticos de Políticas Educativas, 25(48), 1-19. https://doi.org/10.14507/epaa.25.2868

Jiménez, A. y Gutiérrez, A. (2017, noviembre). La calidad educativa en la educación superior: la importancia de su definición por parte de las IES y la evaluación de sus estudiantes al egreso de la licenciatura. Ponencia presentada en el XVI Congreso Nacional de Investigación Educativa, Universidad Autónoma de San Luis Potosí. Recuperado de http://www.comie.org.mx/congreso/memoriaelectronica/v14/doc/2046.pdf

Jiménez, A., Ojeda, D. y Caso, J. (2018). El EGEL desde la perspectiva de los egresados: el caso de la Licenciatura en Administración de la UABC-FCAyS. Debates en Evaluación y Currículum, $4(4)$.

Kappe, R. y Van der Flire, H. (2012). Predicting academic success in higher education: what's more important than being smart? European Journal of Psychology of Education, 27(4), 605-619. https://doi.org/10.1007/s10212-011-0099-9

Krueger, R. A. y Casey, M. A. (2014). Focus groups: A practical guide for applied research. Singapur: Sage publications.

Luna, E. y Rosales, O. (2014). Identificación de las variables de contexto que promueven la calidad de la enseñanza en educación superior. Revista Argentina de Educación Superior, 6(9), 91-109.

Makrinov, N. y Scharager, J. (2005). Situación actual de una muestra de psicólogos egresados de la Pontificia Universidad Católica de Chile. Psykhe, 14(1), 69-77. https://doi.org/10.4067/So7 18-22282005000100006

Martínez, J. y Pérez, J. (2008). Efecto de la trayectoria académica en el desempeño de estudiantes de ingeniería en evaluaciones nacionales. Formación Universitaria, 1(1), 3-12. https://doi.org/10.4067/s0718-50062008000100002

Muñoz, E. (2007). Contexto socioeconómico, percepción del contexto educativo y tiempo de estudio en relación con los resultados de aprendizaje en la educación superior. Innovar, 17(30), 3146 .

Navarro, R. (2003). El rendimiento académico: concepto, investigación y desarrollo. Revista Electrónica Iberoamericana sobre Calidad, Eficacia y Cambio en la Educación, 1(2).

Ocaña, Y. (2011). Variables académicas que influyen en el rendimiento académico de los estudiantes universitarios. Investigación Educativa, 15(27), 165-179. 
Ostrovsky, A. y Di Doménico, C. (2007, agosto). Formación de grado en psicología: opiniones controversiales de profesores argentinos, latinoamericanos y europeos. Comunicación presentada en el XIV Jornadas de Investigación y Tercer Encuentro de Investigadores en Psicología del Mercosur, Universidad de Buenos Aires.

Peniche, R. y Ramón, C. (2018). Desempeño académico y experiencias de estudiantes universitarios mayas en Yucatán, México. Alteridad. Revista de Educación, 13(1).

https://doi.org/10.17163/alt.v13n1.2018.09

Renault, G., Cortada, N. y Castro, A. (2014). Factores que intervienen en el rendimiento académico de los estudiantes de psicología y psicopedagogía. Signos Universitarios, 27(43), 27-34.

Rodríguez, S. y Serrano-García, I. (2017). Práctica de la psicología social-comunitaria en Puerto Rico: voces de sus egresados/as. Revista Puertorriqueña de Psicología, 28(1), 64-78.

Roblero, S. y Orozco, H. (2015). Pronóstico difuso del examen general para el egreso de la licenciatura para ingeniería en computación de la Universidad Autónoma del Estado de México. Research in computing science, 94, 45-58.

Rodríguez, A. y Vieria, M. (2009). La formación en competencias en la universidad: un estudio empírico sobre su tipología. Revista de Investigación Educativa, 27(1), 27-47.

Salazar, M. (2015). Opinión de los empleadores sobre el mercado laboral de los egresados y profesionales en psicología en la Ciudad de La Paz. Estudios en Psicología Social y Laboral, $14,73-94$.

Saldaña, J. (2015). The coding manual for qualitative researchers. San Francisco, CA: Sage.

Sánchez, E. (2007). Contexto socioeconómico, percepción del contexto educativo y tiempo de estudio en relación con los resultados de aprendizaje en la educación superior. Innovar, $17(30), 31-46$

Shulruf, B., Hattie, J. y Tumen, S. (2008). Individual and school factors affecting student's participation and success in higher education. Higher education, 56(5), 613-632. https://doi.org/10.1007/s10734-008-9114-8

Stake, R. (2005). Investigación con estudios de casos. Madrid: Morata.

Tapasco, O., Ruiza, F. y Osorio, D. (2016). Estudio del poder predictivo del puntaje de admisión sobre el desempeño académico universitario. Revista Latinoamericana de Estudios Educativos, $12(2), 148-165$.

Tejedor, J. (2003). Poder explicativo de algunos determinantes del rendimiento en los estudios universitarios. Revista Española de Pedagogía, 61(224), 5-32.

Thomas, D. (2006). A general inductive approach for analyzing qualitative evaluation data. American Journal of Evaluation, 27(2), 237-346.

https://doi.org/10.1177/1098214005283748

Toscano, B., Ponce, J., Margain, M. y Vizcaíno, O. (2016). Estudio exploratorio de los resultados del EGEL-I CENEVAL como base para identificar factores que determinan su acreditación. EducateConCiencia, 9(10), 64-82.

Universidad Autónoma de Baja California. (2006). Estatuto Escolar de la Universidad Autónoma de Baja California. Mexicali: UABC.

Universidad Autónoma de Baja California. (2013). Modelo educativo de la UABC. Mexicali: UABC.

Universidad Autónoma de Baja California. (2015). Plan de Desarrollo Institucional 2015-2019. Mexicali: UABC. 
Universidad Autónoma de Baja California. (2018a). Licenciatura en Psicología, Campus Ensenada y Mexicali. Recuperado de: http://www.uabc.mx/formacionbasica/FichasPE/Lic_en_Psicologia.pdf

Universidad Autónoma de Baja California (2018b). Licenciatura en Psicología, Campus Tijuana. Recuperado de http://www.uabc.mx/formacionbasica/FichasPE/Lic_Psicologia.pdf

Van der Berg, M. y Hofman, W. (2005). Student success in university education: a multimeasurement study of the impact of student and faculty factors on study progress. Higher Education, 5o(3), 413-466. https://doi.org/10.1007/s10734-004-6361-1

Vázquez, J., Hirales, M. y Botello, L. (2017, octubre). El desempeño de candidatos al egreso en los EGEL CENEVAL de la Facultad de Ciencias Humanas de la UABC. Ponencia presentada en el Congreso Debates en Evaluación y Currículum, 3(3).

Villamizar, G. y Romero, L. (2011). Relación entre variables psicosociales y rendimiento académico en estudiantes de primer semestre de psicología. Revista Educación y Desarrollo Social, 1, 4154 .

\section{Breve CV delos autor}

\section{José Alfonso Jiménez Moreno}

Estudió la licenciatura en psicología, es maestro en psicología y doctor en pedagogía por la Universidad Nacional Autónoma de México. Es miembro del Sistema Nacional de Investigadores en el nivel Candidato. Laboró en el Centro Nacional de Evaluación para la Educación Superior (Ceneval) como coordinador del Examen General para el Egreso de la Licenciatura (EGEL). Trabajó como subdirector de pruebas internacionales en el Instituto Nacional para la Evaluación de la Educación (INEE). Actualmente se desempeña como investigador en el Instituto de Investigación y Desarrollo Educativo de la Universidad Autónoma de Baja California. ORCID ID: 0000-0003-0704-7883. Email: jose.alfonso.jimenez.moreno@uabc.edu.mx 